\title{
Research on Optimized Perception of Network Analysis and Evaluation of Communication Access Network
}

\author{
AN Zhi-yuan, LI Wen-cui, WANG Chun-ying, SHU Xin-jian, ZHANG Yong, \\ LUO Zhen
}

Information \& Telecommunication Co. of State Grid Henan Electric Power Company, Zhengzhou 450052, China

Keywords: Access Network, Network Analysis, Optimization Perception

\begin{abstract}
Based on the terminal communication access network evaluation optimization perception technology, completed the terminal communication access network analysis and evaluation optimization perception research. This paper presents a large data based on the network early warning analysis algorithm, develops a large data network early warning analysis model. Then puts forward the intelligent state prediction model and overhauls strategy based on large data, develops the intelligent state maintenance function of large data communication. Finally proposes a network security situation sensing model and evaluation method based on large data, develops a large data network security situational awareness model. According to these methods, we establish a highly efficient, responsive and reliable power terminal communication access network to improve the pre-discovery capability of the hidden trouble of communication network, reduce the accident rate and the seriousness of the accident and improve the quality of distribution network service.
\end{abstract}

\section{Introduction}

With the continuous advancement of smart grid construction of Henan electric power company,we have been building a distribution networksuccessively.Because the distribution network bears many important production of electricity business, such as power distribution automation, telephone services and others, the construction of end to end business management system of power distribution network work increasingly important.

With the rapid development of the business channel, data volume, and the scale of the distribution power communication network, thenetwork structure becomes complicated. This resulted in a large number of communication failures [1]. The current management and monitoring is still heavily dependent on the monitoring personnel's own experience.Therefore, the current network system monitoring largely depends on the network response after the problem occurs, rather than active warning monitoring, seriously affected the communication security and stability of the operation.

At present, the domestic power industry adopts one-way data acquisition mode, which is to collect alarm information, performance information and configuration information through the north directional interface of professional network.The construction of the communication management system by direct affiliated scientific research institutes such as Electric Power Research Institute, have in-depth research and application of the north point interface of mainstream manufacturers, including Huawei, ECI, NEC, Alcatel, Marconi and other equipment manufacturers.

\section{Network Analysis and Evaluation of Access Network}

The terminal communication access network equipment type is complex and the manufacturer type is numerous. Through the analysis of heterogeneous network fault information, the system network is important to protect the site, in the form of warning prompt, make the network maintenance work from passive to active, so as to realize the real-time performance of network operation maintenance, reliability and accuracy. 
The terminal communication access network equipment type is complex, the manufacturer type is numerous [2]. Through to the fragility of heterogeneous network own existence condition, the external environment may lead to the possibility of network security and the possible impact of network risk assessment index system, thus improve the terminal communication access network robustness and stability. Through the analysis of heterogeneous network fault information, the system network is important to protect the site, in the form of warning prompt, make the network maintenance work from passive to active, so as to realize the real-time performance of network operation maintenance, reliability and accuracy.

Network quality optimization is based on the existing operation network, data analysis and field test data acquisition, parameter analysis, hardware inspection and find out the reasons affecting the quality of the network, and through the parameter changes, network structure adjustment, the adjustment of the equipment configuration and some technical means, to ensure the operation of the network quality, make the existing network resources to obtain the best benefit, in the most economic inputs to obtain the biggest benefit [3].

Evaluation module accordingto the terminal access network bandwidth data condition, according to set good parameters, equipment list all over the bandwidth limit, in a different color display, and give treatment advice.

For the equipment that gives the alarm, the system can be classified according to the system, and the light path can be viewed, the real reason of the cable warning can be further examined [4]. It can be associated with the main interface. You can view various alerts in a network topology environment. The results of the analysis are as follows:

Tab.1 Bandwidth analysis table

\begin{tabular}{|c|c|c|c|c|}
\hline Line name & Line type & Bandwidth & Bandwidth utilization (\%) & $\begin{array}{c}\text { Processing } \\
\text { Advice }\end{array}$ \\
\hline $\mathrm{XX}$ & $220 \mathrm{kV}$ & $2.5 \mathrm{G}$ & $80 \%$ & $\begin{array}{c}\text { Network } \\
\text { Optimization }\end{array}$ \\
\hline $\mathrm{XX}$ & $500 \mathrm{kV}$ & $10 \mathrm{G}$ & $85 \%$ & $\begin{array}{c}\text { Adjust the } \\
\text { business path }\end{array}$ \\
\hline
\end{tabular}

The fiber optic analysis and evaluation module can set up the upper limit of the fiber core according to the fiber core of the fiber optic cable, and list all devices that exceed the maximum limit, indicating that the user will be able to make optical cable expansion or modification.

Requirements can be analyzed in real time and can be associated with the main interface. According to the regional classification of the optical cable that gives the alarm, the real reason of the optical cable warning is to be able to see the interface diagram of the optical cable [5]. It can view all the fiber core information of the cable segment, and can check the carrying business information of the occupied fiber core. The results of the analysis are as follows:

Tab.2Fiber optic analysis table

\begin{tabular}{|c|c|c|c|c|c|}
\hline $\begin{array}{c}\text { optical } \\
\text { fiber } \\
\text { name }\end{array}$ & $\begin{array}{c}\text { optical } \\
\text { fiber } \\
\text { type }\end{array}$ & area & $\begin{array}{c}\text { fiber } \\
\text { length }\end{array}$ & $\begin{array}{c}\text { used/all fiber } \\
\text { core number }\end{array}$ & $\begin{array}{c}\text { treatment } \\
\text { recommendations }\end{array}$ \\
\hline XX & OPGW & ZhengZhou & 55 & $22 / 24$ & quit some optical paths \\
\hline XX & ADSS & AnYang & 26 & $20 / 24$ & $\begin{array}{c}\text { adjust the path of the } \\
\text { optical path }\end{array}$ \\
\hline
\end{tabular}

\section{ETA Logic Deduction}

The Event Tree Analysis, ETA, is a logical deduction that analyzes the results of various sequences of events that might be caused by events at a given initial Event. Based on the ETA method, the influence of network expansion on system network can be improved to improve the comprehensiveness and systematization of network impact analysis. The analysis and description method based on graphic model is helpful to the communication and communication of deep people, so as to prevent the quality and efficiency of evaluation due to inexact understanding. Risk assessment method based on the model, which is beneficial to extend relevant elements of access 
network mode abstract and summarized, through the reuse model, as well as the development and application based on the model of tool set, it can improve efficiency, reduce cost, and improve the productivity of risk assessment.

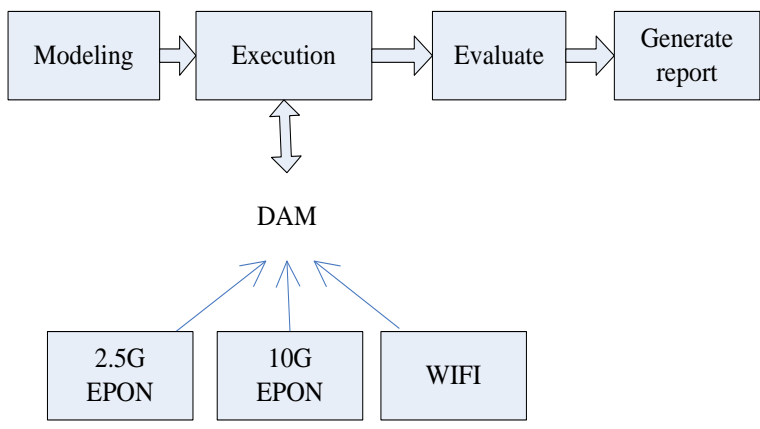

Fig.1 ETA logic deduction process

\section{Modeling PTM and PSM}

The development process of network index comparison analysis and evaluation technique can be divided into three stages: demand analysis, model design and application implementation. In these three stages, two different Modeling processes are included: first, the business model of the system is established during the requirements analysis stage, which is the Platform Independent Modeling (PIM) process. The second is to establish a system model in the design stage, which is the Platform Specific Modeling (PSM) process.

The comparison analysis and evaluation techniques of network index can be summarized in three stages: network analysis, network comparison and application evaluation. Among them, the network analysis and comparison phase describes the system related models using the UML analysis method, which is the continuation and extension of the system demand analysis and system design in the process of network application evaluation. The network recognition stage has the security detection and verification of the system, the identification system faces the threat and the system's vulnerability. Based on the identification phase, the analysis stage is based on the analysis of the related elements such as threat and vulnerability, and establishes the ATA model of key objects. This stage focuses on modeling relevant elements of the system platform. The assessment phase is based on the established model, analysis the key object of network risk probability, the analysis of the effect of damage to the key object security of network, the application of risk assessment key objects node.

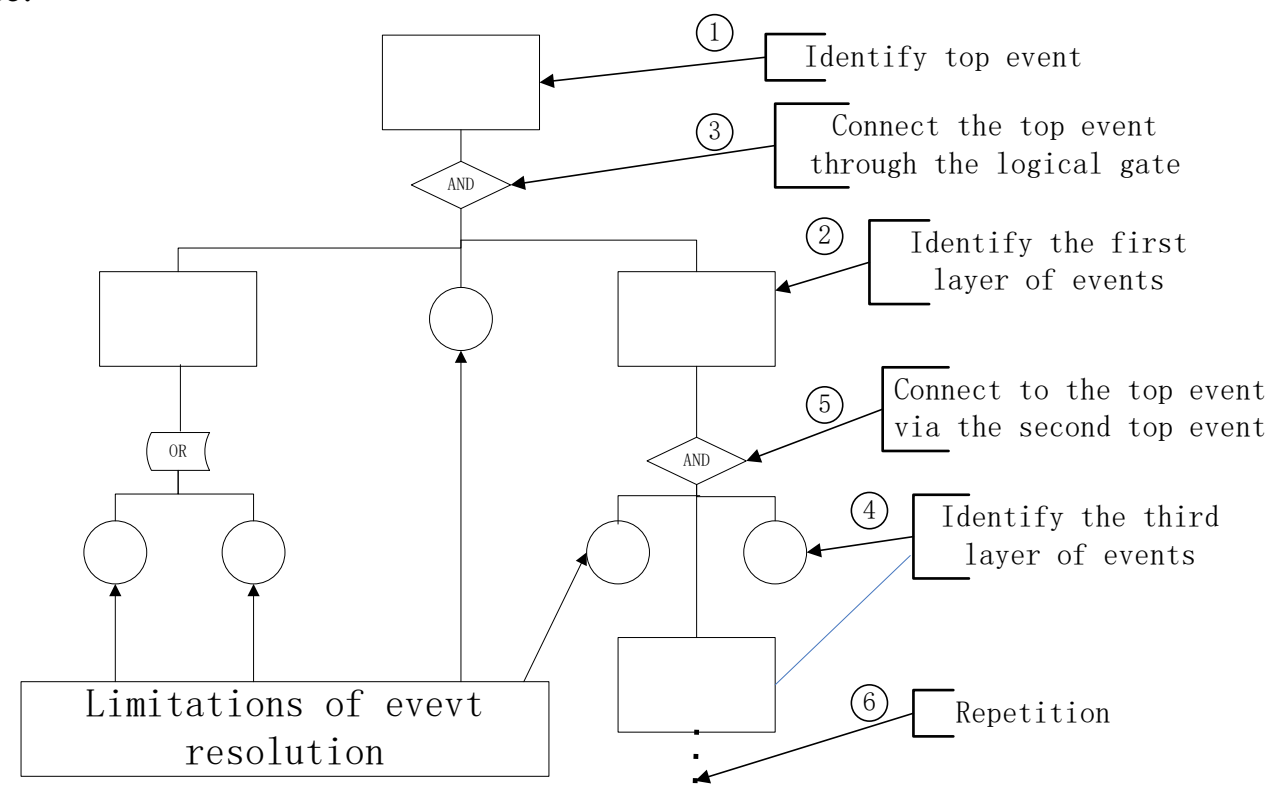

Fig.2 Network analysis and comparison evaluation logic chart 


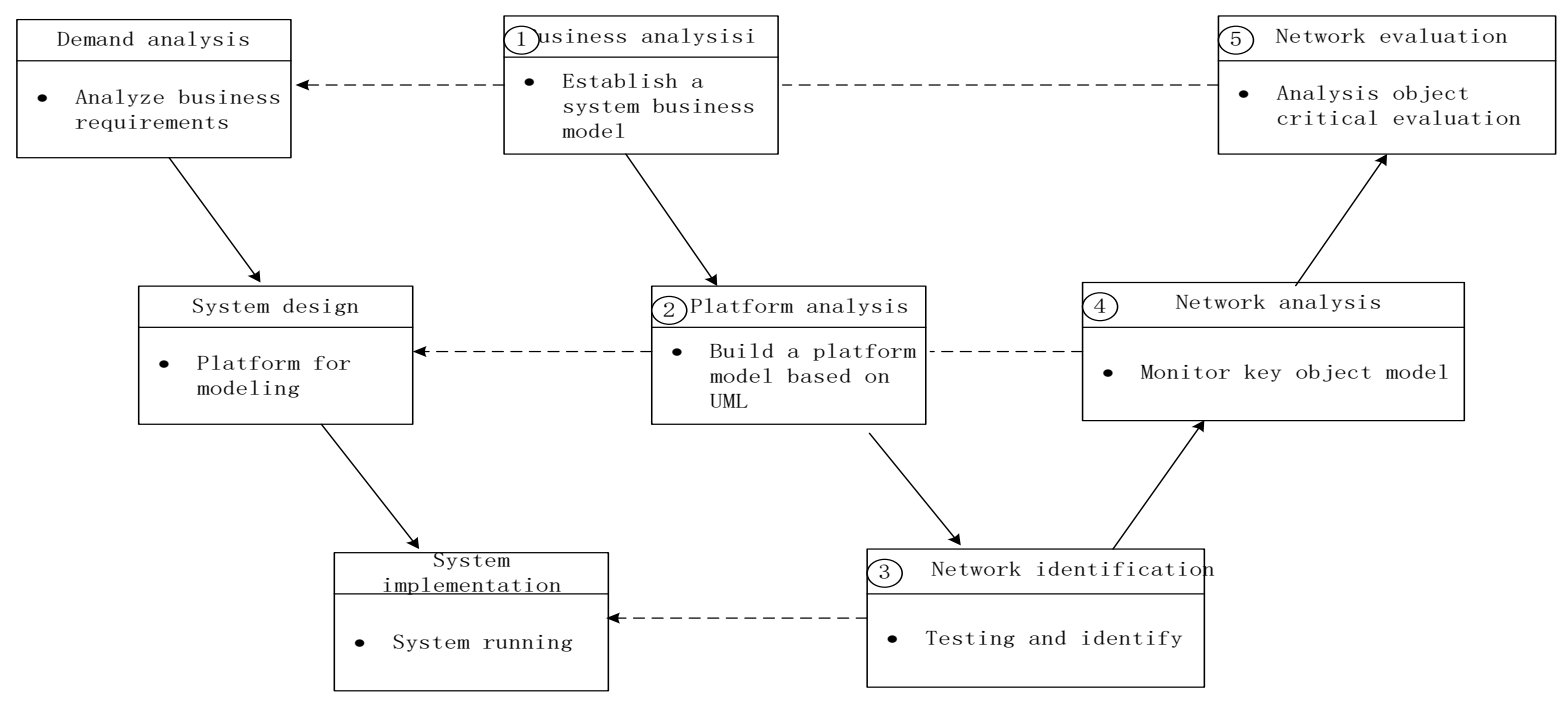

Fig.3 Network analysis comparison evaluation flow chart

\section{Conclusion}

The network analysis and evaluation of terminal communication network analysis and evaluation is an optimization and improvement of the existing network status function of the communication management system of the provincial company. It should be in accordance with the relevant standard specification request, have general, clear, correct format added province communication access network management system model structure, and complete the network early warning analysis, risk assessment, quality optimization and situational awareness of terminal communication network.

\section{References}

[1] Li Wencui, Li Xiong, Zhang Chi, Wu Lijie, ShuXinjian, Tang Weixia. Research on the Figure Module Integration Technology in Communication Monitoring. ICEEECS, 2016(50), 686-689.

[2] LIN Chuan-jie, HE Zhi-yong, ZHU Li-fang. Platform Construction Analysis of Comprehensive Network Resource Management System[J].Digital Technology and Application,2013(1):10-11

[3] Li Wencui, Li Xiong, Yang Ying, Ding Ying , Shu Xinjian, Zhang Yong. A Brief Talk on Information and Communication Safety Management of Electric Power Enterprise. ICMMBE, 2016 (83), 217-220.

[4] Li Wencui, Shu Xinjian, Li Xiong, Gao Hui, Liu Bo, Wang Chunying, Yang Ying. An Information Security Management System Based on "Five-in-one”. ICEEECS, 2016(50), 743-745.

[5] Xu Chunhua, Niu Jigao, Pei Fenglai. Design and simu-lation of the power-train system for an electric vehicle. Artificial Intelligence Management Science and Electronic Commerce . 2011 